\title{
WIND observations of coherent electrostatic waves in the solar wind
}

\author{
A. Mangeney ${ }^{1}$, C. Salem ${ }^{1}$, C. Lacombe ${ }^{1}$, J. -L. Bougeret ${ }^{1}$, C. Perche ${ }^{1}$, R. Manning ${ }^{1}$, P. J. Kellogg ${ }^{2}$, K. Goetz ${ }^{2}$, \\ S. J. Monson ${ }^{2}$, J. -M. Bosqued ${ }^{3}$ \\ ${ }^{1}$ URA 264 du CNRS, DESPA, Observatoire de Paris, 92195 Meudon, France \\ ${ }^{2}$ School of Physics and Astronomy, University of Minnesota, Minneapolis, MN 55454, USA \\ ${ }^{3}$ CESR/CNRS, Université Paul Sabatier, BP 4346, 31029 Toulouse, France
}

Received: 15 June 1998 / revised: 14 September 1998 / Accepted: 15 September 1998

\begin{abstract}
The time domain sampler (TDS) experiment on WIND measures electric and magnetic wave forms with a sampling rate which reaches 120000 points per second. We analyse here observations made in the solar wind near the Lagrange point $L 1$. In the range of frequencies above the proton plasma frequency $f_{p i}$ and smaller than or of the order of the electron plasma frequency $f_{p e}$, TDS observed three kinds of electrostatic (e.s.) waves: coherent wave packets of Langmuir waves with frequencies $f \simeq f_{p e}$, coherent wave packets with frequencies in the ion acoustic range $f_{p i} \leq f<f_{p e}$, and more or less isolated non-sinusoidal spikes lasting less than $1 \mathrm{~ms}$. We confirm that the observed frequency of the low frequency (LF) ion acoustic wave packets is dominated by the Doppler effect: the wavelengths are short, 10 to 50 electron Debye lengths $\lambda_{D}$. The electric field in the isolated electrostatic structures (IES) and in the LF wave packets is more or less aligned with the solar wind magnetic field. Across the IES, which have a spatial width of the order of $\simeq 25 \lambda_{D}$, there is a small but finite electric potential drop, implying an average electric field generally directed away from the Sun. The IES wave forms, which have not been previously reported in the solar wind, are similar, although with a smaller amplitude, to the weak double layers observed in the auroral regions, and to the electrostatic solitary waves observed in other regions in the magnetosphere. We have also studied the solar wind conditions which favour the occurrence of the three kinds of waves: all these e.s. waves are observed more or less continuously in the whole solar wind (except in the densest regions where a parasite prevents the TDS observations). The type (wave packet or IES) of the observed LF waves is mainly determined by the proton temperature and by the direction of the magnetic field, which themselves depend on the latitude of WIND with respect to the heliospheric current sheet.
\end{abstract}

Correspondence to: A. Mangeney
Key words. Interplanetary physics (plasma waves and turbulence; solar wind plasma). Space plasma physics (electrostatic structures).

\section{Introduction}

Electrostatic waves with frequencies $f$ between the ion and electron plasma frequencies $f_{p i} \leq f<f_{p e}$ have been studied for more than 20 years in the inner heliosphere (see the Helios observations and a comparison with the Pioneer observations, Gurnett and Anderson, 1977). Their wavelength $\lambda$ is of the order of a few tens of Debye lengths $\lambda_{D}$ (Gurnett and Frank, 1978), their electric field $\mathrm{E}$ is very closely aligned with the local magnetic field, and the corresponding electric energy density is small compared to the thermal energy density of the plasma: indeed, $\epsilon_{0} E^{2} /\left(2 N \mathrm{k}_{B} T_{e}\right)$ is only about $10^{-7}$ to $10^{-5}$ during intense bursts, and much smaller on the average (Gurnett, 1991). ( $\epsilon_{0}$, vacuum dielectric constant; $N$, particle number density; $k_{B}$, Boltzmann constant; $T_{e}$, temperature of the electrons).

Initially observed with instruments having relatively poor time and frequency resolution, they appeared as very bursty broad band emissions lasting from a few hours to a few days. Observations made with higher time resolution (Kurth et al., 1979) revealed that the emissions were actually formed of brief narrow band bursts with rapidly drifting center frequency.

This electrostatic activity occurs quite frequently but is not a permanent feature of the solar wind. Gurnett et al. (1979) found that the greatest intensity and frequency of occurrence was observed in the slow speed wind where $V_{s w} \leq 500 \mathrm{~km} / \mathrm{s}$ and the electron temperature $T_{e}$ is larger than the proton temperature $T_{p}$. However, substantial wave intensities are also observed for high speed flows and small temperature ratio, $T_{e} / T_{p} \simeq 0.3$. The wave intensity decreases with increas- 
ing distance from the sun and seems to be correlated with the parameters which measure the deviations of the particle distributions from Maxwellian ones (such as the electron heat flux or the quality of the fit of proton distributions by a Maxwellian).

The URAP instrument onboard Ulysses observed waves in the $f_{p i} \leq f<f_{p e}$ frequency band even at high heliographic latitudes. However, the regions with the most intense ion acoustic (IAC) wave activity were found at low heliographic latitudes (MacDowall et al., 1996).

Neither the wave modes nor the source of these waves have yet been unambiguously identified (see Gurnett, 1991). Gurnett and Frank (1978) proposed that they were ion acoustic waves propagating along the ambient magnetic field, with frequencies $f_{0}$ lower than the local ion plasma frequency $f_{p i}$ in the plasma frame, but strongly Doppler shifted to the observed range in the spacecraft frame. Several instabilities have been suggested to be responsible for their occurrence. For example, the electron heat flux (Forslund, 1970; Dum et al., 1981) may be a source of free energy for the e.s. IAC mode. But Gary (1978) has shown that other, electromagnetic, heat flux instabilities have much larger growth rates in conditions typical of the solar wind. Another attractive possibility would be ion beams (Gary, 1978; Lemons et al., 1979; Marsch, 1991) such as those observed more or less continuously in the fast solar wind and drifting with roughly the local Alfvén speed with respect to the core ion distribution. These ion beams could excite IAC waves. However, Gary (1993) concludes that for the range of temperature ratios $T_{e} / T_{p} \leq 5$ usually observed in the solar wind the instability threshold is never reached.

Bursts of Langmuir waves (at $f_{p e}$ ), not related to type III solar bursts, have also been observed in the solar wind (Gurnett and Anderson, 1977; Gurnett et al., 1979); they are frequently associated with magnetic holes (Lin et al., 1995a); their intensity appears to be significantly higher at high heliographic latitudes (i.e. in the fast speed wind) than at low latitudes (MacDowall et al., 1996).

We shall describe here new observations by the time domain sampler (TDS) experiment which is part of the WAVES investigation on the WIND spacecraft. These observations were obtained in the ecliptic plane when WIND was at a distance of the Earth greater than 200 $R_{E}$, near the Lagrange point $L_{1}$. These observations are similar to those analysed by Kurth et al. (1979) but have a higher time resolution, up to 120000 points per second. At this temporal resolution and in the unperturbed (by the Earth) solar wind, the wave forms recorded by the TDS are coherent and display three typical shapes. Two of them correspond to wave forms already known: modulated wave packets at the Langmuir frequency, and modulated wave packets with centre frequencies $f$ in the IAC wave range $\left(f_{p i} \leq f<f_{p e}\right)$ which will be called the low frequency (LF) range in the following. The third type of wave form, which has not been previously reported in the free solar wind, consists of isolated electrostatic structures (IES) similar in many respects to those observed in different regions of the Earth's environment (Temerin et al., 1982, Matsumoto et al., 1994, Mottez et al., 1997). We shall also discuss some observations indicating that this last type of wave form occurs in the vicinity of the L1 Lagrange point for all solar wind conditions, while the frequency of occurrence of the LF wave packets decreases with increasing distance from the heliospheric current sheet.

\section{The data}

The WAVES experiment on the WIND spacecraft measures the radio and plasma waves in a large range of frequencies, from a fraction of a Hertz up to $14 \mathrm{MHz}$ for the electric field, and up to $3 \mathrm{kHz}$ for the magnetic field (Bougeret et al., 1995).

In this study, we use the electric and magnetic wave form measurements from the time domain sampler (TDS) which is part of the WAVES package. This instrument uses two orthogonal antennas, the $x$-antenna, a wire dipole of physical length $2 \times L_{x}\left(L_{x}=50 \mathrm{~m}\right)$ tip-to-tip, and the $y$-antenna, a much shorter dipole of physical length $2 \times L_{y}\left(L_{y}=7.5 \mathrm{~m}\right)$ tip-to-tip. To obtain the electric field $E$ along the $x$ or $y$ antenna, we divide the measured electric potential difference $V$ at the antenna terminals by the corresponding effective electric lengths $\bar{L}_{x}$ or $\bar{L}_{y}$. These effective electric lengths differ from the physical ones due to the effects of the spacecraft environment; they are known within a few percent, $\bar{L}_{x}=41.3 \mathrm{~m}$ and $\bar{L}_{y} \simeq 0.1 \bar{L}_{x}$. Since the signal-to-noise ratio is usually smaller by an order of magnitude on the short $y$-antenna than on the $x$-antenna we shall mainly use the data obtained with the $x$-antenna, except in Sect. 4.

The electromagnetic waveforms are organised as time series of 2048 data points, which will be called events in what follows. To remain within the telemetry constraints, only a small number of the events collected are transmitted. The selection criteria can be modified, but in any case only the events with a peak amplitude above a programmable noise threshold are collected. We have analysed in detail a limited period, from May 20 to June 26, 1995, when WIND was at more than $200 R_{E}$ from the Earth. During this period (and until March 16, 1996) the additional criterion for transmission was the following: each time the telemetry was available to the TDS, the most recently recorded event was transmitted, so that roughly 100 to 300 events per day are available, with an average of one event every $10 \mathrm{~min}$.

Note that some of these events are seriously affected by parasitic signals, twice per spin. The corresponding signals are generally weak but, as the receiver is logarithmic, these weak signals may be above the programmable noise threshold (the threshold corresponds to an instantaneous peak amplitude of $\simeq 50 \mu \mathrm{V} /$ $\mathrm{m}$ and to an integrated square electric field of $10^{-11}$ $\mathrm{V}^{2} \mathrm{~m}^{-2}$ ). These signals are impulses apparently generated by some experiment or spacecraft system which are coupled through the plasma to the antenna, since their apparent amplitude increases with the plasma density. 
In dense regions (typically when $N \geq 12 \mathrm{~cm}^{-3}$ ), the parasitic signal is above the threshold and, as it occurs every $1.5 \mathrm{~s}$, it is transmitted in place of less frequent natural signals.

Three sampling rates were used for the measurements of the electric wave forms, 120000 sample per seconds (sps) every two days and either 30000 sps or 7500 sps every other day. The duration of the corresponding records is respectively 17, 68 or 273 milliseconds. Note that the highest sampling rate resolves wave forms at the electron plasma frequency $f_{p e}$, and sometimes at its harmonic $2 f_{p e}$ (Kellogg et al., 1996; Bale et al., 1996). Magnetic wave forms were measured only with the lowest sampling rate $(7500 \mathrm{sps})$.

To allow the statistical analysis of the following sections we have been led to characterise quantitatively and on a routine basis the intensity and type of the wave form seen by the TDS, using a small number of parameters. The first parameters are three spectral parameters. They are related to the shape of the coefficients of the Fourier series of the electric potential $\hat{V}(f)$ (in volts), as a function of $f$, for each event (2048 points): a weighted central frequency $f_{m}$,

$f_{m}=\frac{\Sigma[f\|\hat{V}(f)\|]}{\Sigma\|\hat{V}(f)\|}$

a bandwidth $\Delta f$,

$\Delta f=\left[\frac{\Sigma\left[\|\hat{V}(f)\|\left(f-f_{m}\right)^{2}\right]}{\Sigma\|\hat{V}(f)\|}\right]^{1 / 2}$

and a mean square electric field $\overline{E^{2}}$ (in $\mathrm{V}^{2} \mathrm{~m}^{-2}$ ) taking into account the antenna length $L_{x}$ :

$\bar{E}^{2}=2 \Sigma\|\hat{V}(f)\|^{2} / L_{x}^{2}$.

The sums are taken over the frequencies for which $\|\hat{V}(f)\|$ is greater than one tenth of its maximum value $\|\hat{V}\|_{\max }$, in order to limit the effects of the wide band noise. In what follows, we shall use $\bar{E}^{2}$ as a measure of the electric energy density $\epsilon_{0} \bar{E}^{2} / 2$.

The artefacts mentioned have an easily recognisable spectrum limited to the frequency band [0-400] Hz. Therefore if the electric energy density contained in the spectrum above $400 \mathrm{~Hz}$ is smaller than a certain threshold, the event will be considered as an artefact; otherwise it will be considered as a natural signal.

For the period considered here, there is a well-defined gap in frequency around $12 \mathrm{kHz}$ between the high frequency Langmuir waves (and harmonics) and the low frequency IAC waves. The treatment is therefore made separately on each frequency band $f<12 \mathrm{kHz}$ and $f>$ $12 \mathrm{kHz}$. In the high frequency band, due to the frequent presence of harmonics of $f_{p e}$, the high frequency limit of the summation in Eqs. (1) to (3) is restricted to 1.5 times $f_{p e}$. Note that this last range does not exist for the lowest sampling rates.

Another parameter characterises the way the electric energy is distributed in time during the event. We have introduced the intermittence of each event i.e. the ratio of the numbers of points with a voltage below and above a given threshold $S$ among the 2048 points of each event. More precisely, let $N_{1}$ be the number of points $i$ for which $|V(i)-\langle V(i)\rangle| \leq S$ and $N_{2}$ the number of points for which $|V(i)-\langle V(\bar{i})\rangle|>S$, so that $N_{1}+N_{2}=2048$. The intermittence is then defined by

$I=N_{1} / N_{2}$.

A threshold of $S=8 \times 10^{-4} \mathrm{~V}$ has been chosen empirically to provide the best separation between typical low frequency wave forms. Note that the value of the intermittence parameter depends on the sampling rate; in what follows it has been calculated only for the highest one.

The local plasma parameters which we have used for comparison were obtained as follows. The electron density comes from a neural network (Meetre et al., 1998; Richaume et al., 1995). This determination of the electron density $N_{e}$ relies on the identification of the local electron plasma frequency, $f_{p e}=\left[N_{e} q^{2} /\left(\epsilon_{0} m\right)\right]^{1 / 2} / 2 \pi$ ( $m$ and $q$ being the electron mass and charge) in the spectrum of the thermal noise measured between 4 and $246 \mathrm{kHz}$ by the TNR receiver (Meyer-Vernet and Perche, 1989; Bougeret et al., 1995). The uncertainty on the neural network density $N_{e}$ is usually below $5 \%$ although it can reach $15 \%$ when the density falls below $5 \mathrm{~cm}^{-3}$.

The other solar wind properties which have been used here, the bulk velocity $\mathbf{V}_{s w}$, the electron and proton temperatures $T_{e}$ and $T_{p}$, and magnetic field data come from the key parameters file (KP), have a low time resolution of $1.5 \mathrm{~min}$ and are respectively given by the 3D-plasma experiment (Lin et al., 1995b) and the MFI experiment (Lepping et al., 1995).

To close this section, we add some definitions: $M$ is the proton mass, $v_{\text {the }}=\sqrt{\left(k_{B} T_{e} / m\right)}$ the electron thermal speed, and $\lambda_{D}=\left[\epsilon_{0} k_{B} T_{e} /\left(N_{e} q^{2}\right)\right]^{1 / 2}$ is the electron Debye length.

\section{The three types of electrostatic waves}

The most prominent result of the TDS observations in the free solar wind is that, above the proton plasma frequency, the electromagnetic waves are essentially coherent and made of a succession or a combination of only three types of wave forms.

To illustrate this property we have displayed in Fig. 1 six typical TDS events observed on different days. Figure 1a is a good example of the first type of wave form, namely modulated high frequency Langmuir wave packets, oscillating at the local electron plasma frequency, $f_{p e} \simeq 18 \mathrm{kHz}$. Its appearance and its amplitude $\left(\Delta V_{x} \simeq 0.01 \mathrm{~V}\right.$ at the antenna terminals i.e. an electric field of $\simeq 0.2 \mathrm{mV} / \mathrm{m}$ ) are similar to the weakest events observed in the Earth foreshock (Bale et al., 1997).

Figure 1b, c displays two examples of the second type of wave form which are low frequency wave packets. The first one (Fig. 1b) is a narrow band signal with a centre frequency $f \simeq 3.4 \mathrm{kHz}\left(f_{p e} \simeq 17 \mathrm{kHz}\right)$. The second one (Fig. 1c) is also a narrow band sinusoidal wave 

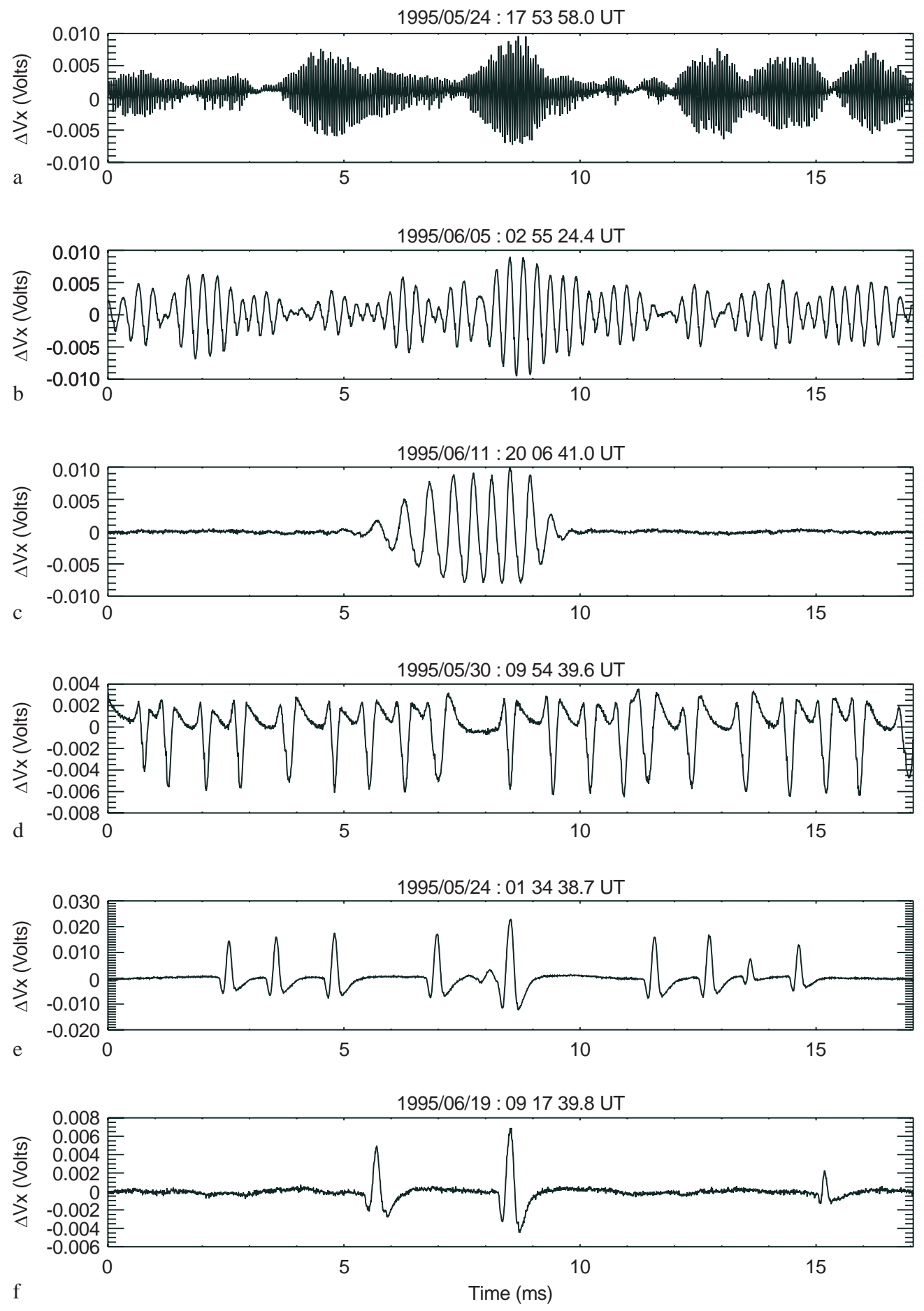

Fig. 1a-f. Six typical wave forms observed by the TDS in the solar wind. The electric potential $\Delta V_{x}$ in volts is measured at the terminals of the $x$ antenna, $L_{x}=50 \mathrm{~m}$. a Langmuir waves; $\mathbf{b}$ and $\mathbf{c}$ low frequency quasi-sinusoidal wave packets; d, e and $\mathbf{f}$ non sinusoidal wave packets and isolated electrostatic structures (IES)

at a frequency $f \simeq 2.4 \mathrm{kHz},\left(f_{p e} \simeq 18 \mathrm{kHz}\right)$ but with a much shorter envelope of temporal width $\simeq 5 \mathrm{~ms}$. In both cases the maximum electric field is of the order of $0.1 \mathrm{mV} / \mathrm{m}$, close to the values observed for the IAC waves on Helios 1 by Gurnett and Anderson (1977). The wave form of Fig. 1d is nearly periodical at $2.4 \mathrm{kHz}$ $\left(f_{p e} \simeq 25 \mathrm{kHz}\right)$ but it has a strongly non-sinusoidal appearance, indicating the presence of significant nonlinear effects. Most probably, the narrow band bursts with a variable centre frequency observed by Kurth et al.
(1979) were LF wave packets similar to the one shown in Fig. 1c.

Finally, Fig. 1e, f displays two examples of the third type of wave form, which has not yet been reported in the solar wind. They are isolated spikes of duration 0.3 to 1 millisecond with amplitudes similar to those of the wave packets just described. We call them isolated electrostatic structures (IES). Note that the Helios receiver has probably not detected the IES because of its relatively long integration time $(50 \mathrm{~ms}$, Gurnett and 
Anderson, 1977). In Fig. 1e, f the IES are clearly isolated, whereas in Fig. 1d the spikes are so closely packed that the resulting wave form does not appear very different from the modulated wave packet of Fig. $1 b$.

No correlation has been noted between the orientation of the spacecraft with respect to the Sun and the presence of IES. Therefore these waves are not likely to be artefacts due to the variable illumination of the spacecraft or of the electric antennas.

The spikes are very similar in shape and duration to the electric pulses parallel to the magnetic field observed in the auroral acceleration regions and identified as double layers (Temerin et al., 1982; Mozer and Temerin, 1983; Boström et al., 1988). They may also be compared with the electrostatic solitary waves ESW which have been detected in the plasma sheet boundary layer in the magnetosphere (Matsumoto et al., 1994; Kojima et al., 1994). These ESW appear as the well-known broad band electrostatic noise (BEN) when observed in the frequency domain. However the most frequently observed ESW are bipolar pulses: they are made of two successive electric pulses of roughly equal amplitude but of opposite sign; this shape is different to that shown in Fig. 1f.

With the spectral parameters introduced (Eqs. 1 to 3) the distinction between Langmuir wave packets and LF wave packets is easily made since their central frequencies belong to well-separated frequency bands.

To illustrate how the spectral parameters and the intermittence $I$ (Eq. 4) allow us to separate the two types of low frequency waveforms, Fig. 2a displays a scatter plot of $I$ as a function of $\Delta f / f_{m}$ for all the TDS events (2753) observed at $f_{m}<12 \mathrm{kHz}$. In this plane, four regions have been delimited and two of them can be clearly identified. Since IES are limited in time, they have a broad band Fourier spectrum and a large intermittence: $\Delta f / f_{m}=0.5$ for the wave forms shown in Fig. 1e, f while $I \simeq 2$ (Fig. 1e) and $I \simeq 9$ ( Fig. 1f). Thus IES are found in region III of Fig. 2a, $\Delta f / f_{m}>0.35$ and $I>1.5$. The LF wave packets are more sinusoidal and correspond to region I, $\Delta f / f_{m}<0.4$ and $I \leq 1.2$; indeed they cover a narrower frequency band and have a weaker intermittence. For example, $\Delta f / f_{m}=0.1$ and $I=0.3$ in Fig. $1 \mathrm{~b}$, the corresponding values being 0.3 and 4 in Fig. 1c. Region II corresponds to wave forms which are not easily classified in one or the other category of wave forms, as for example the almost periodic succession of IES of Fig. 1d, for which $\Delta f / f_{m}=0.5$ and $I=0.6$. Finally, region IV corresponds to very short wave packets such as the one displayed in Fig. 1c.

Note that the Langmuir wave events have a very small relative band width and a small intermittence: for the wave form of Fig. $1 \mathrm{a}, \Delta f / f_{m}=0.017$ and $I=0.65$.

Figure $2 \mathrm{~b}$ displays the integrated energy density $\bar{E}^{2}$ (Eq. 3, in $\mathrm{V}^{2} \mathrm{~m}^{-2}$ ) of the LF e.s. events as a function of their relative band width $\Delta f / f_{m}$. The range is $3 \times 10^{-11}$ to $3 \times 10^{-7} \mathrm{~V}^{2} \mathrm{~m}^{-2}$ (the Langmuir waves, not shown, have the same energy density range). $E^{2}$, integrated during the $17 \mathrm{~ms}$ of each event, decreases when $\Delta f / f_{m}$
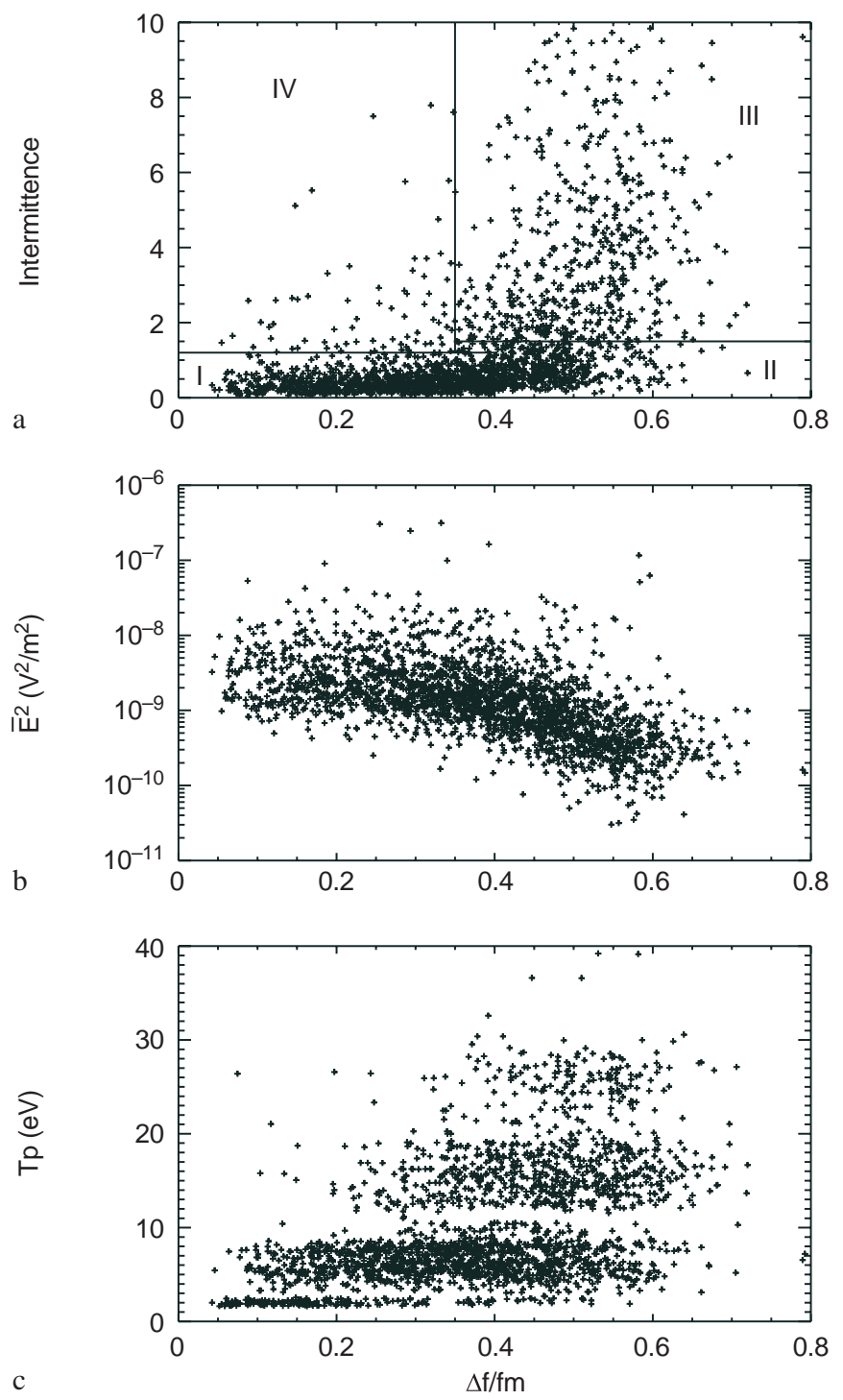

Fig. 2a. Scatter plot of the intermittence $I$ (Eq. 4) versus the relative band width, for all the LF events observed with the highest sampling rate. LF wave packets are found in region I, and isolated electrostatic structures in region III. b Scatter plot of the energy density, in $\mathrm{V}^{2} / \mathrm{m}^{2}$, versus the relative band width for the events of a. c scatter plot of the solar wind proton temperature $T_{p}$ in $\mathrm{eV}$, at the time of the e.s. events of a versus the relative band width of the events

increases: IES, which have the same amplitude as wave packets (Fig. 1), have a lower energy density because they are more intermittent (Fig. 2a).

It is also interesting to note that the proton temperature $T_{p}$ in the solar wind appears to be correlated with the spectral properties of the LF wave activity. This is illustrated by Fig. 2c which displays a scatter plot in the plane $\left[\Delta f / f_{m}, T_{p}\right]$ for the TDS events. A comparison of Fig. $2 \mathrm{~b}$ and $\mathrm{c}$ indicates that the events observed when $T_{p} \geq 10 \mathrm{eV}$ have a relatively broad band $\left(\Delta f / f_{m} \geq 0.3\right)$ and thus carry a weaker energy density $\bar{E}^{2}$. We have not found any correlation between the electron temperature and $\Delta f / f_{m}$ or $E^{2}$. In Sect. 6 , we shall discuss in more details the correlations between the wave properties and the solar wind parameters. 


\section{Properties of the low frequency wave packets}

We shall now study the properties of the low frequency wave packets seen by the TDS and show that they have, besides their frequency range, all the properties of the IAC waves as described for example in the review by Gurnett (1991).

\subsection{Polarisation}

We first note that, when the magnetic and electric wave forms were available, at the lowest sampling rate used here, no significant magnetic counterpart was observed, indicating that in the range of frequency $f_{p i} \leq f \leq f_{p e}$ the waves are basically electrostatic.

On the other hand, the TDS provides simultaneous measurements $E_{x}$ and $E_{y}$ of the electric field amplitude on the two orthogonal $x$ and $y$ antennas. These measurements may be used to obtain the polarisation of the waves and some information about the direction of the electric field in the plane of the two antennas. Indeed, if $\Delta V_{x}$ and $\Delta V_{y}$ are the potential differences measured respectively at the terminals of the $x$ and $y$ antenna, their ratio $r_{x y}=\Delta V_{y} / \Delta V_{x}$ is a function of the angle between the electric field $\mathbf{E}$ and the unit vector along the $x$-antenna, $\mathbf{a}_{x}$. However, since the $y$-antenna is much shorter than the $x$-antenna, the corresponding signal-to-noise ratio is much lower; so that in order to measure $r_{x y}$ the only reliable method is to perform a linear regression $\Delta V_{y}=\alpha \Delta V_{x}+\beta$ and to assume that the coefficient $\alpha$ is a good estimate for $r_{x y}$. The results are shown in Fig. 3, which display a scatter plot of the antenna ratio $r_{x y}$ versus the cosine of the angle $\theta_{x B}$ between $\mathbf{a}_{x}$ and the magnetic field $\mathbf{B}$. In Fig. 3a, $r_{x y}$ is shown for the wave packets and in Fig. $3 b$ for the IES. It is clear that $r_{x y}$ tends to decrease when $\theta_{x B}$ decreases, as expected if $\mathbf{E}$ is aligned with $\mathbf{B}$. More precisely, let us calculate the ratio $\bar{r}_{x y}$ obtained by assuming a triangular distribution of current along the antennas and a monochromatic wave with a wave vector $\mathrm{k} L_{x}=1.1$, typical of the wave packets, as we shall see in Sect. 4.2. Let us also assume that the wave vector $\mathbf{k}$ is aligned with $\mathbf{B}$, so that $\theta_{x k}=\theta_{x B}$. The expression for $\Delta V_{x}$ is then

$\Delta V_{x} \propto \bar{L}_{x} \cos \left(\theta_{x B}\right)\left[\frac{\left.\sin \left[\left(k L_{x} \cos \theta_{x B}\right) / 2\right)\right]}{\left(k L_{x} \cos \theta_{x B}\right) / 2}\right]^{2}$

and a similar expression for $\Delta V_{y}$. The theoretical ratio $\bar{r}_{x y}$ calculated in this way is the function of $\theta_{x B}$ shown in Fig. 3a (solid line) for $\bar{L}_{y} / \bar{L}_{x} \simeq 0.1$. It is equal to zero for $\theta_{x B}=0$ (the $y$-antenna is insensitive to electric fields aligned with the $x$-antenna), it increases as $\theta_{x B}$ increases towards $\pi / 2$, and it exhibits a divergence at $\theta_{x B} \simeq \pi / 2$ (the $x$-antenna is insensitive to electric fields aligned with the $y$-antenna). For small angles the theoretical curve is compatible with the data, indicating that the antennas behave as expected. However, the divergence of the $r_{x y}$ data expected at large angles is not seen, a fact which may be explained in several ways. First, only TDS
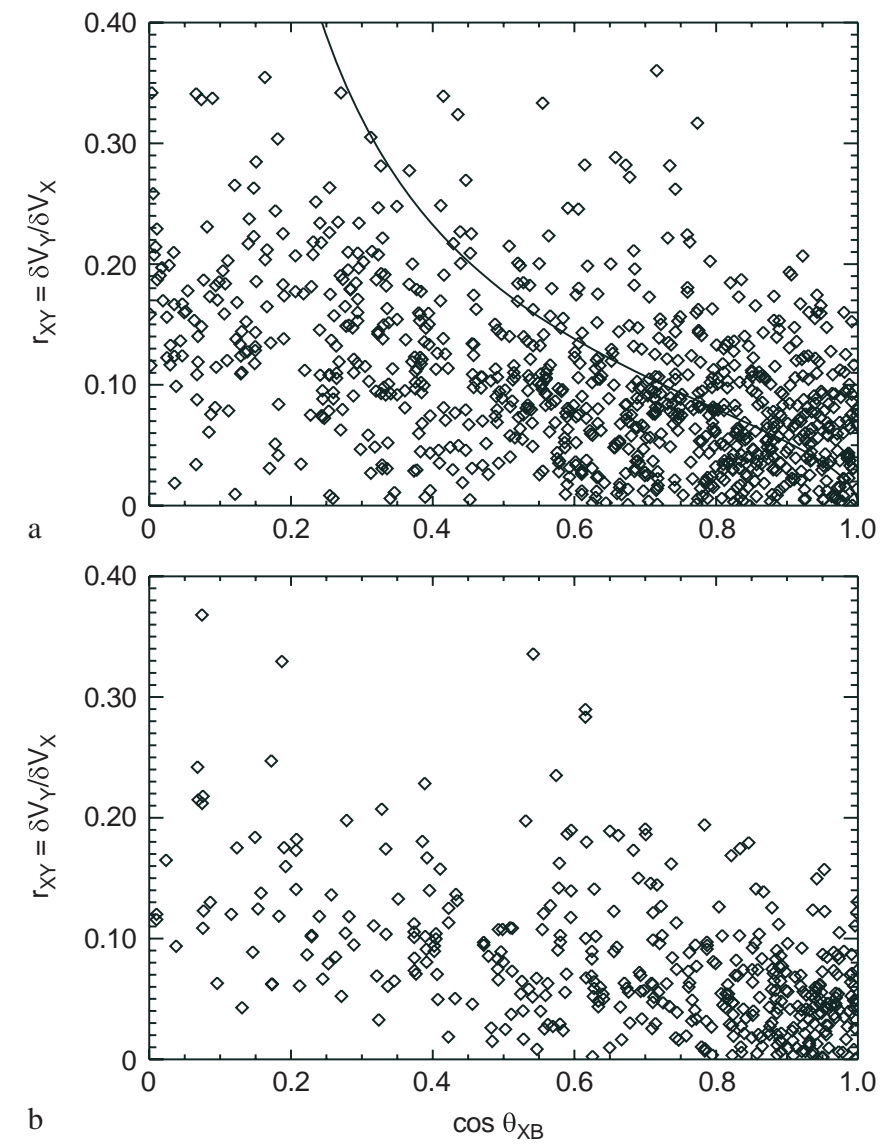

Fig. 3a, b. Scatter plot of the ratio $r_{x y}$ of the voltage differences $\Delta V_{y}$ and $\Delta V_{x}$ measured respectively on the $y$ and $x$ antenna versus the cosine of the angle $\theta_{x B}$ between the magnetic field and the direction of the $x$-antenna a for wave packets; the solid line is a theoretical ratio $\bar{r}_{x y}$ based on Eq. (5) for $\mathrm{k} L_{x}=1.1$; b For IES

events where the electric field on the $x$-antenna is above a certain threshold are collected, so that very large values of $r_{x y}$ are not expected. Second, since we use interpolated key parameter file magnetic field data, our estimate of the angle $\theta_{x B}$ is probably not good enough, especially close to $\pi / 2$. Furthermore, due to the presence of the spacecraft, the antennas may not behave exactly as orthogonal electric dipoles aligned with the physical antennas.

A similar trend is observed for the IES (see Fig. 3b), the ratio $r_{x y}$ also increasing with $\theta_{x B}$. A theoretical prediction of $r_{x y}$ is however much harder to calculate for IES without a model of the original (non sinusoidal) waveform in the plasma. Nevertheless, two conclusions may be drawn. First, the fact that the ratio $r_{x y}$ between the output of the two antennas is correlated with the angle $\theta_{x B}$ between the local magnetic field and the $x$ antenna reinforces the argument of Sect. 3: the observed signals are not parasites (such as the shadow artefact described) but are produced in the solar wind plasma. Second, our results are consistent with the hypothesis that the wave vector is more or less aligned with the magnetic field, for IES as well as for the LF wave packets. The use of magnetic field data with a higher temporal resolution could help to be more specific. 


\subsection{Doppler effect and wavelength}

Let us now analyse of the Doppler effect on the observed frequencies. Consider first the LF wave packets and let $f_{0}$ be their frequency in the plasma rest frame, $\mathbf{k}$ their wave vector ( $\lambda=2 \pi / k$ being the corresponding wavelength) and $f$ their frequency in the spacecraft frame. Since the waves are propagating along the local magnetic field direction $\mathbf{b}, \mathbf{k}=k \mathbf{b}$, the relation between $f_{0}$ and $f$ can be written as .

$\frac{f}{f_{p i}}=\frac{f_{0}}{f_{p i}}+k \lambda_{D} \frac{\Delta f_{\text {Dopp }}}{f_{p i}}$

where

$\frac{\Delta f_{\text {Dopp }}}{f_{p i}}=\left(\frac{M}{m}\right)^{1 / 2} \frac{V_{s w} \cos \theta_{B V}}{v_{\text {the }}}$

is the Doppler contribution to the observed frequency when $k \lambda_{D}=1$, and $\theta_{B V}$ is the angle between the solar wind velocity and $\mathbf{b}$.

Figure $4 \mathrm{a}$ shows a scatter plot of the relative frequency $f_{m} / f_{p i}$ versus $2 \pi \Delta f_{\text {Dopp }} / f_{p i}$ for the LF wave packets observed by the TDS, during the 19 days with the highest sampling rate, and selected as described in
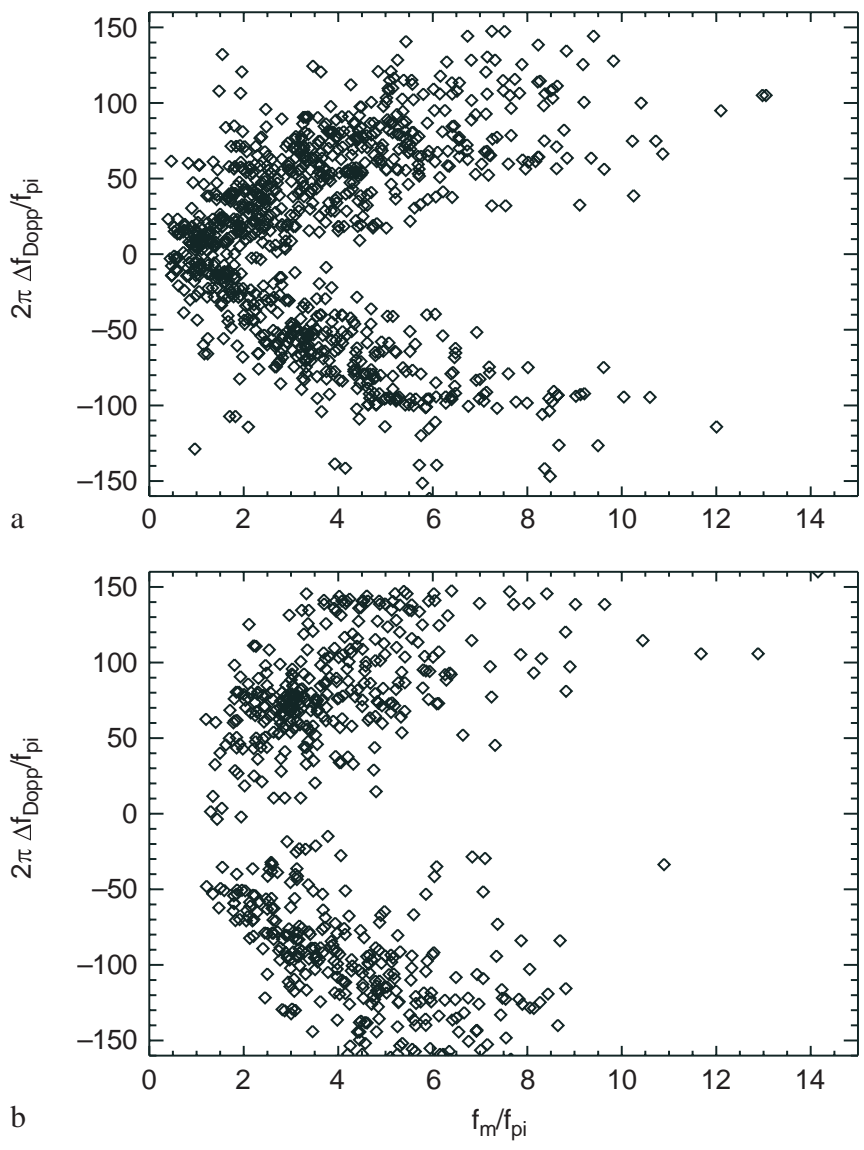

Fig. 4a, b. Scatter plot of the Doppler frequency (Eq. 7) versus the centre frequency $f_{m}$ (these frequencies are normalised to the local proton plasma frequency $f_{p i}$ calculated with the TNR density). a For the LF wave packets (region I in Fig. 2a); b for the isolated electrostatic structures (region III in Fig. 2a)
Sect. 3 (region I of Fig. 2a). The cloud of points is Vshaped with a tip located around $\cos \theta_{B V} \simeq 0$ and $f_{m} / f_{p i} \leq 1$ and two linear branches, one for each sign of $\cos \theta_{B V}$. This result can be understood on the basis of Eq. 6 if the main contribution to the observed frequencies comes from the Doppler effect, thus confirming the previous analysis by Gurnett and Frank (1978). It is also consistent with the results obtained already that the waves are propagating along the ambient $\mathbf{B}$ field. It shows further that the wave numbers of the wave packets do not cover a wide range. By estimating the slopes of the straight lines between which most of the points of Fig. 4a can be found, we find

$$
10 \leq \frac{\lambda}{\lambda_{D}} \leq 50 \text { or } 0.1 \leq k \lambda_{D} \leq 0.6 \text {. }
$$

Note that this estimate of the range of wave numbers of the LF wave packets is actually an upper limit, part of the scatter in Fig. 4a being probably due to the use of 1.5 min resolution magnetic and plasma data.

These results are compatible with the usual dispersion relation of the ion acoustic waves; indeed, if we assume that the wave packets obey the linear, low frequency, dispersion relation

$\frac{f_{0}}{f_{p i}}=k \lambda_{D}\left(1+\frac{3 T_{p}}{T_{e}}\right)^{1 / 2}$

we find that the frequency in the plasma frame lies typically in the interval $0.1 \leq f_{0} / f_{p i} \leq 2$ which is both the range of values observed in Fig. $4 \mathrm{a}$ when the magnetic field is nearly perpendicular to the solar wind velocity, and the typical range of ion acoustic waves. It has been suggested (Marsch, 1985) that waves observed in the range $f_{p i}<f<f_{p e}$, in the Earth's foreshock, were electron acoustic waves supported by the two electron populations (core and halo electrons). But the electron acoustic waves have a rest frame frequency higher than $f_{p i}$, for parameters usually found in the solar wind, i.e. higher than $f_{0}$ deduced from Fig. $4 \mathrm{a}$.

We note in Fig. 4a that no wave packet has a frequency (in the spacecraft frame) lower than $\simeq 0.3 f_{p i}$ $(\simeq 200 \mathrm{~Hz})$, and this minimum frequency is obtained when $\cos \theta_{B V} \simeq 0$. Therefore the Doppler effect always increases the frequency, independently of the sign of the radial component of the magnetic field. This shows that the wave vector i.e. the direction of propagation of the observed wave packet, even if aligned with $\mathbf{B}$, is oriented towards the Earth. It is unlikely that this result could be due to an observational bias. First, the total duration ( $\simeq 17 \mathrm{~ms})$ of a TDS event at the highest sampling rate allows to resolve wave packets with frequencies of the order of $60 \mathrm{~Hz}$. Second, the threshold above which an event is retained for an eventual transmission by the TDS experiment depends on the frequency, being about $\simeq 50 \mu \mathrm{V} / \mathrm{m}$ at a few $\mathrm{kHz}$, and significantly higher, $\simeq 200 \mu \mathrm{V} / \mathrm{m}$, below $500 \mathrm{~Hz}$, in order to reject more efficiently low frequency aliases. However these instrumental limitations cannot explain why a significant number of wave packets with relative frequency $f_{m} / f_{p i} \geq 0.3$ were observed, and none below. 
To summarise this section, the waves measured by the TDS are electrostatic waves, with a wavelength of a few tens of Debye lengths propagating along the local magnetic field. In the plasma frame they have a low frequency $f_{0} \leq f_{p i}$, which is shifted upwards by the Doppler effect into the range $f_{p i} \leq f<f_{p e}$ in the spacecraft frame. Therefore we have established firmly the relation between the LF wave forms observed by TDS and the electrostatic activity known as IAC waves. In conclusion, the observational evidence accumulated in the past and with the TDS experiment indicates that the IAC waves are coherent wave packets propagating along the ambient $\mathbf{B}$ field, with wave numbers and frequencies compatible with the dispersion relation of ion acoustic waves.

\section{Properties of the isolated electrostatic structures}

IES similar to the ones shown in Fig. 1e, f are frequently observed; in many cases, the LF wave packets look like a rapid, nearly periodic succession of individual IES (Fig. 1d).

\subsection{Doppler effect and spatial width}

To estimate the spatial extension of the IES on a statistically significant sample, we have used a method similar to the one used to analyse the Doppler effect on the LF wave packets. Indeed, for a non periodic signal which is limited in time, the mean frequency $f_{m}$ is proportional to the inverse of the duration $\Delta t$ of the signal, $f_{m} \simeq 1 / \Delta t$. This has been checked on a limited sample of events which we have analysed in detail, with the expected result that the relation is true when the TDS event contains only one or very few spikes. Therefore, it is possible to use the spectral characteristics described in Sect. 3. The spatial extent along $\mathbf{B}$ of an IES, normalised to the local Debye length, can be estimated as

$\frac{\Delta x}{\lambda_{D}} \simeq 2 \pi \frac{f_{p e}}{f_{m}} \frac{V_{s w} \cos \theta_{V B}}{v_{t h e}}$

if the IES velocity in the solar wind frame is negligible.

We shall limit ourselves here to the events belonging to region III of Fig. 2a, i.e. with sufficiently large relative band width and intermittence, $\Delta f / f_{m}>0.35, I>1.5$ : this region only contains TDS events made of a small number of IES so as to minimise the aliasing produced by the presence of several IES in the same TDS event. For those IES, Fig. $4 \mathrm{~b}$ shows the scatter plot of $f_{m} / f_{p i}$ versus $\Delta f_{\text {Dopp }} / f_{p i}$ (Eq. 7). Once again we obtain a cloud of points which is roughly V-shaped, showing that the main part of the observed temporal width of an IES is due to the advection by the solar wind of the structure past the spacecraft. This shape confirms the inference that the IES are indeed propagating along the magnetic field, as do the wave packets, with a speed which is small compared to the solar wind velocity.
Figure 5 displays the corresponding histogram of spatial widths of the IES (Eq. 10), a typical value being $\simeq 25 \lambda_{D}$. Once again it is hard to decide whether the width of the histogram is real or results from the use of low resolution magnetic field and plasma parameters.

\subsection{Potential jump}

Assuming that the IES are one-dimensional structures varying only along the magnetic field, we can easily determine the spatial profiles of the corresponding electric field, electric potential $\Phi$ and charge density $\delta q$. To obtain the sign of the electric field we must take into account the orientation of the antenna $\mathbf{a}_{x}$ with respect to the solar wind velocity, measured by $\cos \theta_{x V}=\mathbf{a}_{x} \cdot \mathbf{V}_{s w} / V_{s w}$.

Let us call $E$ the electric field along the magnetic field; it is related to the voltage $\Delta V_{x}$ measured at the $x$-antenna terminals by the relation

$E(t)=-\Delta V_{x} \operatorname{sign}\left(\cos \theta_{x V}\right) /\left(\bar{L}_{x}\left|\cos \theta_{x B}\right|\right)$.

with the convention that it is positive if directed towards the Earth i.e. along $V_{s w}$. This electric field $E(t)$ is shown in Fig. 6a for the largest IES of Fig. 1f. We have only considered here a finite time interval $\left[t_{1}, t_{2}\right]$ of duration $\simeq 4.2 \mathrm{~ms}$ and containing $\simeq 500$ points around the time of maximum. This interval has been chosen so that it was possible to define unambiguously two asymptotic states where the field is almost constant and equal to $E_{\infty}$ which has been taken as the zero of the electric field. To eliminate the high frequency noise of Fig. 6a, the time profile has been smoothed over 10 points in the region of rapid variation and set to zero outside; the resulting electric field profile is shown in Fig. 6b. The potential $\Phi$ is calculated from the definition as

$\Phi(t)=V_{s w}\left|\cos \theta_{V B}\right| \int_{t_{1}}^{t} \mathrm{~d} \tau\left[E(\tau)-E_{\infty}\right]$

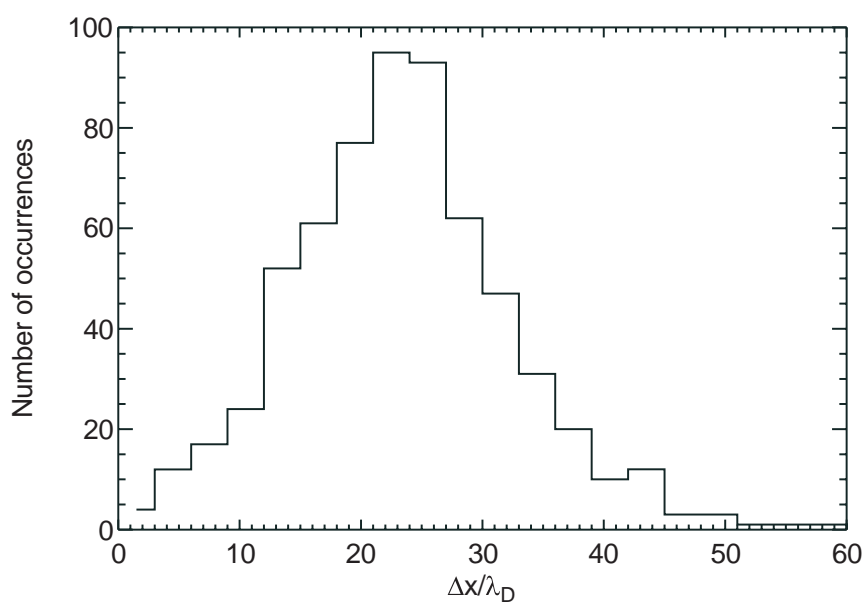

Fig. 5. Histogram of the spatial width of the 626 IES which belong to region III in Fig. 2a 

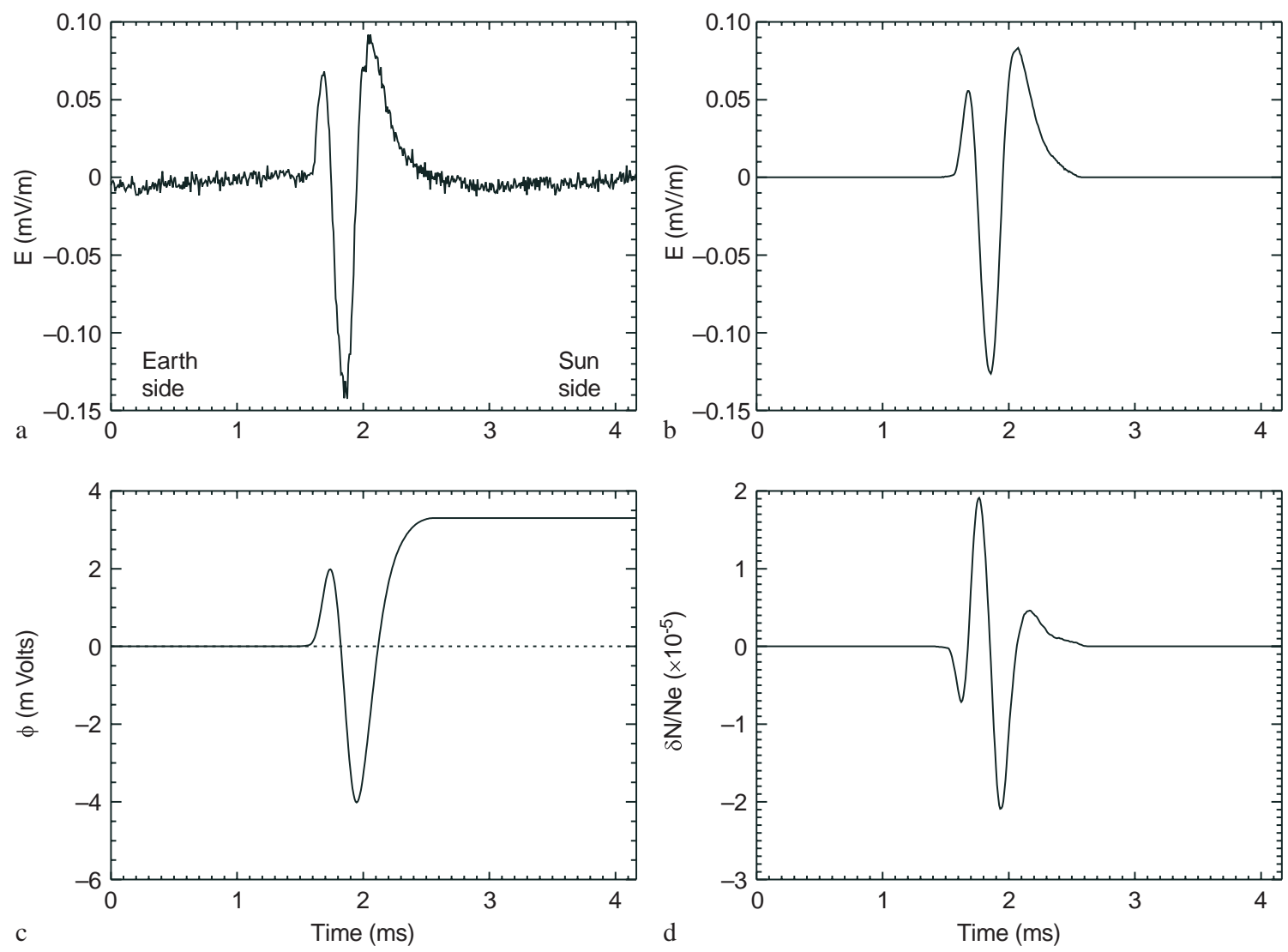

Fig. 6a-d. For the central double layer in Fig. If (between 8 and $9 \mathrm{~ms}$ ), time profile of: a the measured electric field (positive if directed towards the Earth); b the electric field smoothed over 10 points; c the corresponding potential profile; $\mathbf{d}$ the charge separation $\delta N=N_{p}-N_{e}$

while the charge separation $\delta N=N_{p}-N_{e}$ is obtained from Poisson's equation

$\delta N(t)=-\frac{1}{4 \pi q V_{s w}\left|\cos \theta_{V B}\right|} \frac{\mathrm{d} E}{\mathrm{~d} t}$.

The potential profile is shown in Fig. 6c. The remarkable feature is a finite potential jump of $\Delta \Phi \simeq 3.3 \mathrm{mV}$ between the two asymptotic states, the low potential side being towards the Earth. Since $\Delta \Phi$ is proportional to the average value $\langle E\rangle$ of the electric field over the interval $\left[t_{1}, t_{2}\right]$, the estimate of the potential jump is very sensitive to the various offsets which may affect the data and which we have supposed to be included in the nonzero value of $E_{\infty}$. However it can be checked that, in absolute value, $\langle E\rangle$ is significantly higher than $E_{\infty}$, so that our estimate of the potential jump is not seriously affected.

We have repeated the calculation for a significant number (75) of IES which have been chosen so that (1) they are clearly isolated structures (obtained by selecting TDS events of sufficiently high intermittence $I \geq 5$ ) and (2) the geometry is simple. By the latter we mean that the acute angles between the directions of the $x$-antenna, of B and of the solar wind velocity are all smaller than $45^{\circ}$. The results were similar to those shown in Fig. 6. Figure 7 displays the histogram of the normalised potential jumps $q \Delta \Phi /\left(k_{B} T_{e}\right)$ measured for these 75 IES; a typical value is $q \Delta \Phi /\left(k_{B} T_{e}\right) \simeq 3 \times 10^{-4}$.

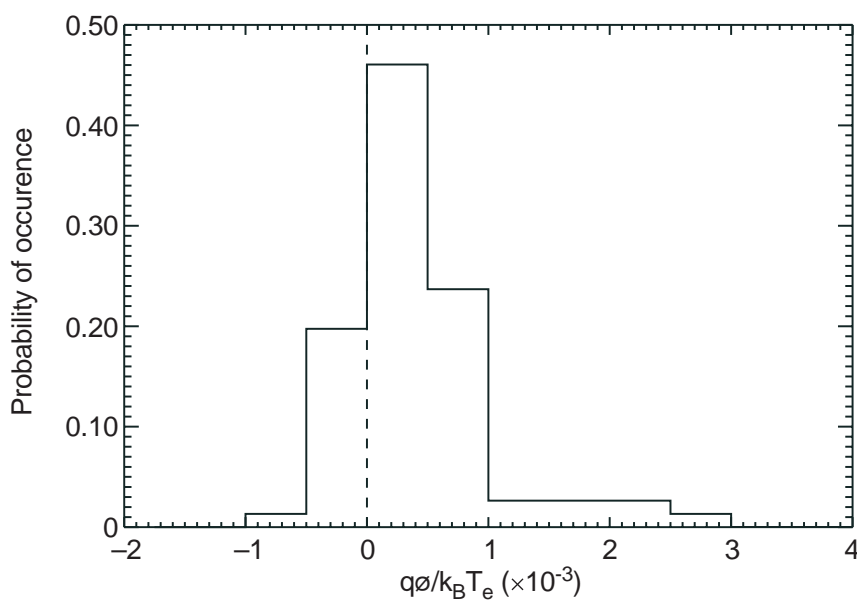

Fig. 7. Histogram of the normalised potential jump for 75 weak double layers selected as described in Sect. 5.2

Therefore, the isolated electrostatic structures are actually weak double layers (WDL) with small relative amplitudes. They consist (see Fig. 6d) of two main layers of approximatively opposite charge, the positively charged one usually preceding the negatively charged one. Note that besides these two layers, one may distinguish a small negative precursor and a positive trailing region. The corresponding density fluctuation $\delta N / N$ is very small $\simeq 10^{-5}$. The similarity with the WDL observed in the auroral regions (Mozer and Temerin, 
$1983)$ is striking although there the peak amplitudes reach much higher values, $q \Delta \Phi /\left(k_{B} T_{e}\right) \simeq 1$. We have also calculated the average electric field and the potential jump across short wave packets for which the asymptotic levels are clearly defined and, surprisingly, found a result not very different from the typical potential jumps across IES. For example, across the wave packet of Fig. 1c, the potential jump is $q \Delta \Phi /\left(k_{B} T_{e}\right)=2 \times 10^{-4}$. For the 75 weak double layers in the sample described, the potential jump (as a function of time) has generally the same sign as $\cos \theta_{x V}$. Therefore, this potential usually drops towards the Earth: it varies in the same sense as the interplanetary electric potential which tends to decelerate the solar wind outward propagating electrons, or to accelerate the protons. The weak double layers, as well as some wave packets, probably manifest a small scale charge separation due to a partial decoupling between electrons and protons on a Debye length scale. We have thus now to look for the solar wind regions where the electrostatic activity has been observed, and for the regions where the weak double layers are dominant.

\section{Solar wind properties and electrostatic activity}

We shall consider the question of how the electrostatic activity observed by the WIND/WAVES experiment depends on the local parameters of the solar wind. The 38-day period considered here is well suited for such an investigation since it exhibits a large range of variations of the physical properties of the solar wind. WIND was more then $85 \%$ of the time in the free solar wind i.e. not magnetically connected to the Earth's bow shock. It crossed three high speed flows, two in the Northern magnetic field Hemisphere, one in the Southern Hemisphere (Sanderson et al., 1998) and spent a long interval of time in a relatively low speed wind, in both hemispheres (Fig. 8). Six main crossings of the magnetic sector boundary were observed (days 142-143, 150, 158$159,165,168-170$ and 176) as well as four reverse interplanetary shocks (days 144, 150, 170 and 177). Unfortunately, however, natural electrostatic activity is observed by the TDS experiment only in regions of the solar wind where the particle density is below $N \leq 12$ $\mathrm{cm}^{-3}$, because of the artefacts discussed in Sect. 2. As a consequence, we were not able to make a complete statistical study of the occurrence of the IAC wave activity. Nevertheless, some interesting results have been obtained, which we shall now describe.

\subsection{Synoptic view of the TDS observations}

In Fig. 8, the electrostatic wave activity as observed by the TDS experiment is displayed as a function of time for two successive intervals of 19 days. Figure 8a covers the period from day $1995 / 05 / 20$ (day 140) to $1995 / 06 / 07$ (day 158), while Fig. 8 b covers that from day 1995/06/08 (day 159) to 1995/06/26 (day 177). In the upper panels, the upper line gives the solar wind speed and the other line the ratio $T_{p} / T_{e}$. In the lower panels, the central frequency and the band width (Eqs. 1 and 2) of every TDS event are indicated by a vertical bar centred on $f_{m}$ with an extent of $f_{m} \pm 0.2 \Delta f$. To illustrate the limitations imposed on the TDS observations, time intervals during which only artefacts are transmitted are indicated in Fig. 8a, b by bars at $30 \mathrm{~Hz}$ : this is the case, for example, on the days $159 / 95 / 06 / 08$ and $169 / 95 / 06 / 18$ (see Fig. 8b). The electron plasma frequency $f_{p e} \geq 12$ $\mathrm{kHz}$ and gyrofrequency $f_{c e} \simeq 100 \mathrm{~Hz}$ are given in each figure, as well as the Nyquist frequencies $(3.8,15$ and 60 $\mathrm{kHz}$ ) for the three sampling rates.

The e.s. activity occurs in two well-separated frequency bands, the LF band significantly below the local electron plasma frequency $f_{p e}$, and a narrow band around $f_{p e}$. In the high frequency range, our method eliminates the waves at harmonics of $f_{p e}$ except in a few ambiguous cases. With these restrictions the Langmuir waves have a frequency $f_{m}$ which follows very closely the local $f_{p e}$, confirming the mode identification. Since $f_{p e}$ is generally higher than $15 \mathrm{kHz}$, Langmuir waves can only be observed every two days, for the highest sampling rate. At the intermediate sampling rate (days 155 or 171 for example) aliasing of Langmuir waves are sometimes observed around $10 \mathrm{kHz}$.

Taking into account the limitations of the TDS observations, no apparent correlation can be found between the occurrence of the TDS activity and the plasma parameters displayed in Fig. 8, the solar wind speed and electron to proton temperature ratio: TDS events are observed on the day $151\left(V_{s w}>700 \mathrm{~km} / \mathrm{s}\right.$, $\left.T_{p} / T_{e}>2\right)$ as well as on the day $164\left(V_{s w} \simeq 300 \mathrm{~km} / \mathrm{s}\right.$, $\left.T_{p} / T_{e}<0.5\right)$.

This is emphasised in Fig. 9: a 2D histogram of the values of $\left[V_{s w}, T_{e} / T_{p}\right]$ at all the times sampled by the TDS is displayed as a one level contour line. The data fill a relatively narrow region of the plane, illustrating the well-known correlation between $V_{s w}$ and $T_{p}$ : fast winds have higher proton temperatures than slow winds, the electron temperature having a smaller range of variation. The energy density $\bar{E}^{2}$ of the $17 \mathrm{~ms}$ TDS events (calculated using Eq. 3) is also shown as contours with grey levels in Fig. 9. It appears that the $17 \mathrm{~ms}$ TDS events have been observed for almost all solar wind conditions (except, of course, during periods of high density). The distribution of energy density $\bar{E}^{2}$ is almost uniform in the $\left[V_{s w}, T_{e} / T_{p}\right]$ plane. There is however a weak but significant trend towards smaller energies in faster winds (with weaker $T_{e} / T_{p}$ ). This trend is in agreement with the Helios results (Fig. 5, 7 and 8 of Gurnett et al., 1979).

6.2 Correlation between the properties of the electrostatic waves and the solar wind parameters

It seems worthwhile to look now for the solar wind parameters which correlate with the energy density, the band width and the type of LF wave forms. We use here the sample of TDS events showing 985 low frequency wave packets (region I of Fig. 2a) and 626 

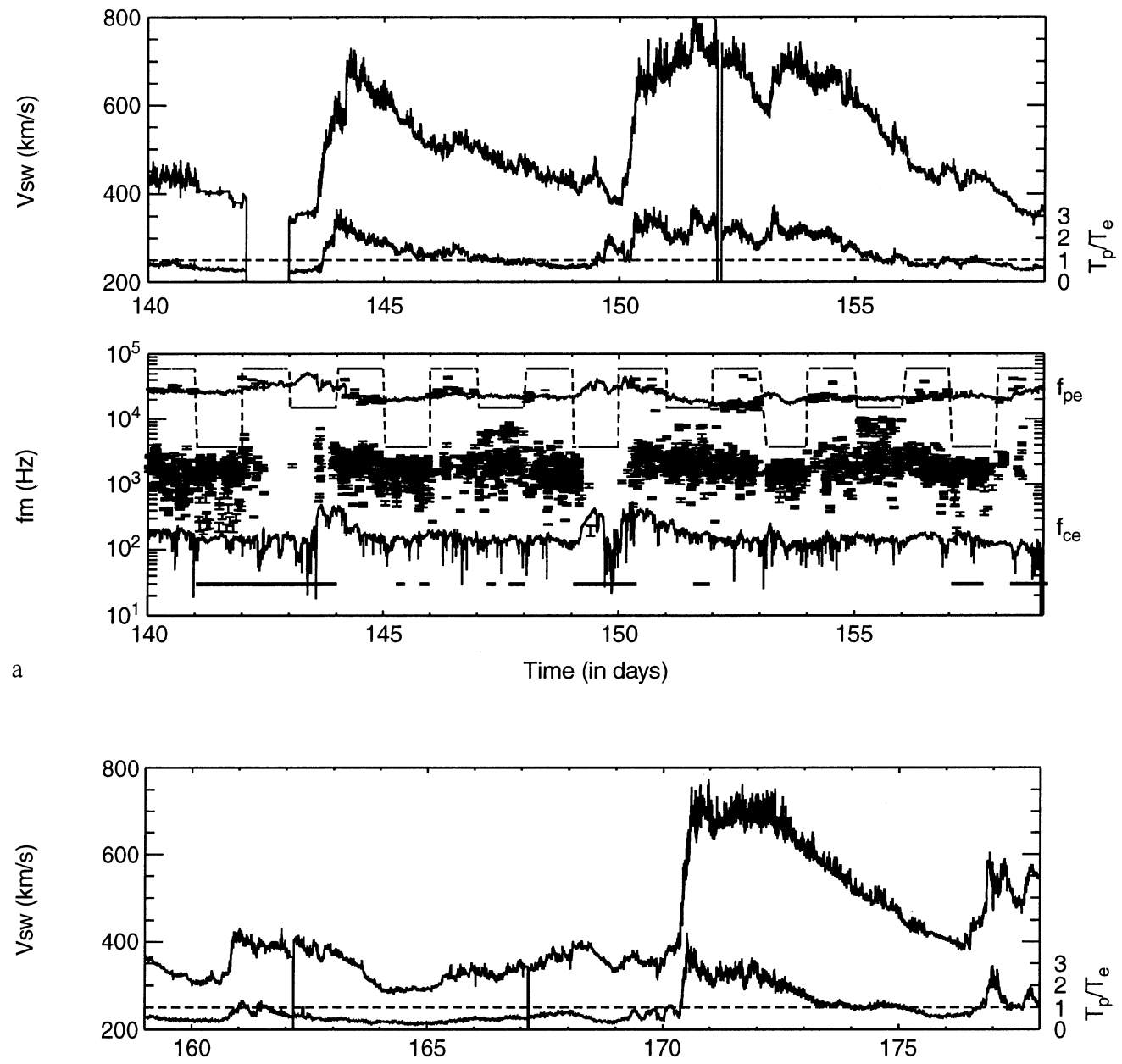

Fig. 8a, b. Synopsis of the solar wind properties and e.s. activity: a from May 20 to June 7, 1995. The numbers in abscissae indicate the beginning of the corresponding day of the year. Upper panel: $V_{s w}$ and $T_{p} / T_{e}$. Lower panel: each TDS event is indicated by a small vertical bar at its frequency $f_{m} \pm 0.2 \Delta f$ (see Eqs. 1 and 2). The characteristic frequencies $f_{p e}$ (3D-plasma data) and $f_{c e}$ are also given. b The same, from June 8 to 26,1995

b

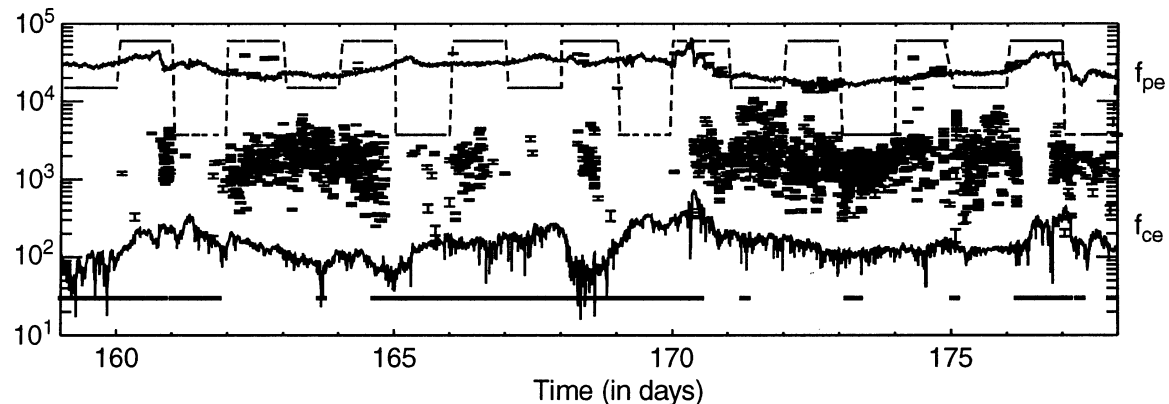

We have already shown (Sect. 3, Fig. 2b, c) that the weak double layers (region III of Fig. 2a) during the 19 days of the period studied here with the highest sampling rate.

We have found two parameters, namely the angle $\theta_{B X}$ between the magnetic field and the Earth-Sun direction $\mathbf{e}_{X}$ and the proton temperature $T_{p}$, which appear to organise the data satisfactorily.

This is illustrated in Fig. 10a, b. Figure 10a displays the histogram of wave packets (dashed line), and WDL (solid line) in equal bins of $\left|B_{X} / B\right|$ : the probability of occurrence of wave packets does not depend on the modulus of $\cos \theta_{B X}$, while the probability of WDL events has a clear minimum for $\mathbf{B}$ perpendicular to $\mathbf{e}_{X}$, and peaks for $\mathbf{B}$ along the Parker spiral and for more radial fields. events observed for $T_{p} \geq 10$ or $11 \mathrm{eV}$ have a relatively broad band $\Delta f / f_{m} \geq 0.3$ and a weaker energy density $\bar{E}^{2}$. The scatter plot of Fig. 2 c shows that $\Delta f / f_{m}$ tends to increase with $T_{p}$. To illuminate the interpretation of the scatter plot of Fig. 2c, Fig. 10b displays histograms of $T_{p}$ measured at the time of LF wave packets (dashed line) and of weak double layers (solid line). Crudely speaking, the wave packets and the WDL are both present when $T_{p}<10 \mathrm{eV}$, while for higher proton temperatures $T_{p}>$ $12 \mathrm{eV}$ the WDL are dominant. Thus, the wave packets disappear gradually when $T_{p}$ increases from 10 to $30 \mathrm{eV}$. Since there is a good correlation between $T_{p}$ and $V_{s w}$ this disappearence of the wave packets is also observed for $V_{s w}>500 \mathrm{~km} / \mathrm{s}$. This is probably the reason for the 


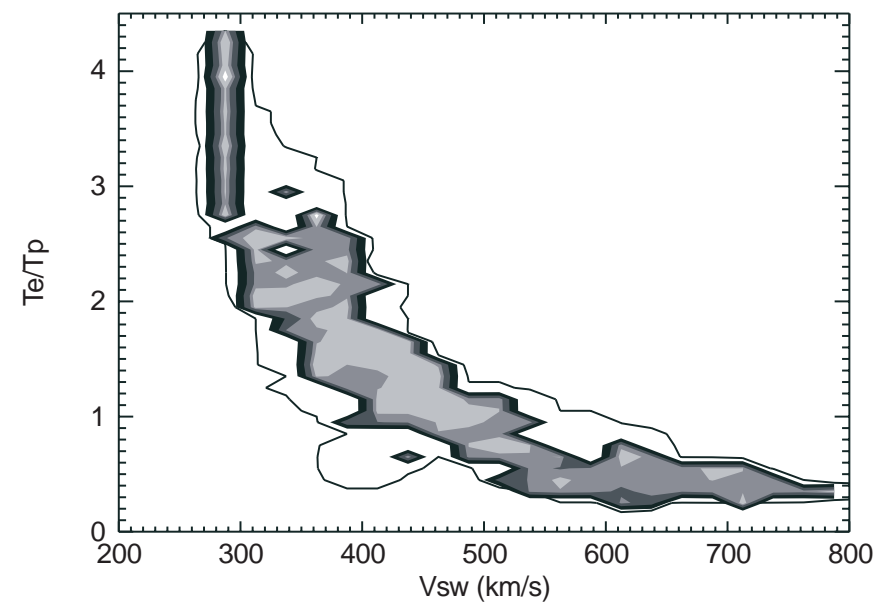

Fig. 9 The thin line delimits the region of the plane $\left[V_{s w}, T_{e} / T_{p}\right]$ sampled by the TDS. The grey contours give the energy density $E^{2}$ of the $17 \mathrm{~ms}$ TDS events, calculated with Eq. 3. The five grey levels correspond to the values $-12,-10.5,-9.35,-8.75$ and -7.75 for $\log _{10} E^{2}$, in $\mathrm{V}^{2} / \mathrm{m}^{2}$

decrease of the electrostatic energy with increasing solar wind velocity mentioned in Sect. 6.1.

One would have expected that the temperature ratio $T_{p} / T_{e}$, which is related to the damping of ion acoustic waves, would also be correlated to the occurrence of wave packets. Indeed, the gradual disappearance of wave packets occurs for $T_{p} / T_{e} \geq 1.2$. However, a scatter plot (not shown here) similar to Fig. 2c but for $T_{p} / T_{e}$, although exhibiting the same trend towards an increase of $\Delta f / f_{m}$ with increasing $T_{p} / T_{e}$, does so in a less convincing way, just as one would expect if $T_{e}$ was a statistically unrelated variable, with a range of variation 6 to 7 times smaller than that of $T_{p}$. There is thus no evidence that a local macroscopic parameter like the temperature ratio $T_{e} / T_{p}$ plays a significant role for the type of the wave forms, contrary to theoretical expec-

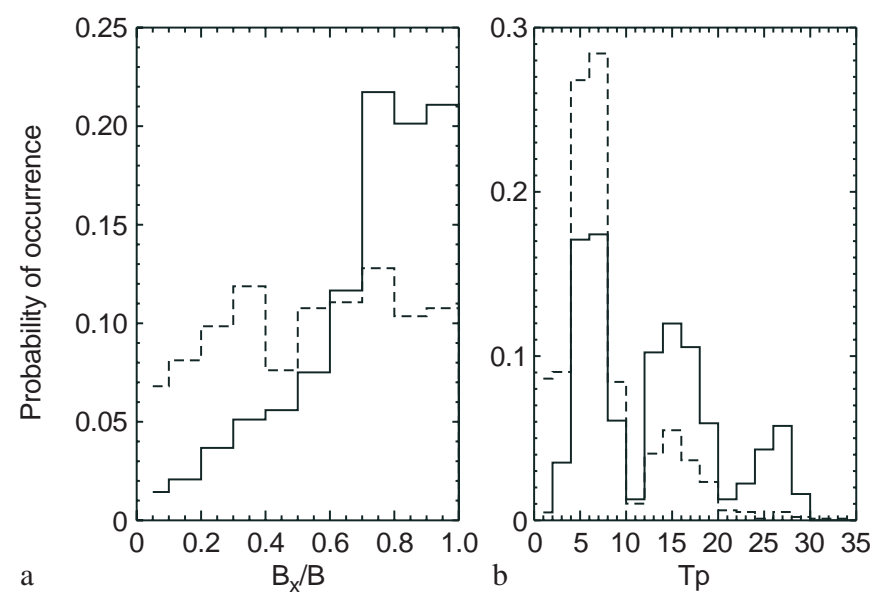

Fig. 10a, b. Histograms of the probability of occurrence of the IES (region III of Fig. 2a; solid line) and wave packets (region I of Fig. 2a, dashed line) as functions: a of the modulus $\left|B_{X} / B\right|$ of the cosine of the angle between the $\mathbf{B}$ field and the Earth-Sun direction. b Of the proton temperature in $\mathrm{eV}$. Each histogram is normalised to its total number of events tations and observations in other regions of the Earth environment (Kojima et al., 1994) and numerical simulations (Omura et al., 1994, 1996).

We conclude from Fig. 10 that isolated electrostatic structures, i.e. weak double layers, are found for any value of $T_{p}$ but preferably when the field is close to the radial. The LF wave packets, i.e. TDS events with more sinusoidal wave forms are preferably found for low values of $T_{p}$, independently of the field direction.

\section{Discussion}

It was totally unexpected to find weak double layers in the free solar wind. These double layers are very weak, compared with those observed in numerical simulations (see Borovsky, 1984), in laboratory plasmas (see Fälthammar, 1993), or in the auroral regions (Mälkki et al., 1993) where typically $q \Delta \phi \simeq k_{B} T$. The whole LF e.s. activity is only very weakly nonlinear, if the main source of nonlinearity is related to the macroscopic kinetic pressure, as is usually the case in the theoretical analysis of nonlinear ion acoustic waves (see e.g. Treumann and Baumjohann, 1997). Indeed, consider the equation of motion of the proton fluid:

$\frac{\partial \mathbf{u}}{\partial t}+\mathbf{u} \cdot \nabla \mathbf{u}=-\frac{q}{M} \nabla \phi+\cdots$.

In the linear approximation, $\delta u$ being the velocity fluctuation and $c_{s}$ the sound speed, this gives

$\frac{\partial \delta u}{\partial t} \simeq-c_{s} \frac{\partial \delta u}{\partial x} \simeq \frac{q}{M} \frac{\partial \phi}{\partial x}$.

The degree of nonlinearity, i.e. the ratio between the nonlinear term in the left hand side of Eq. (14) and the electric restoring force, can be estimated as the Mach number $\delta u / c_{s} \simeq q \Delta \Phi / k_{B} T_{e}$, which is generally weaker than $10^{-3}$ for LF wave packets as well as for WDL, as seen in Sect. 5.2. If we assume further that these fluctuations are weakly nonlinear ion acoustic waves with a typical wave vector $\mathrm{k}$, their group velocity is of order $v_{g} \simeq c_{s}\left[1-(3 / 2) k^{2} \lambda_{D}^{2}\right]$. We can then define a typical time for the dispersal of a wave packet of width $\Delta x \simeq 2 \pi / k$ via dispersive effects, $\tau_{d} \simeq \Delta x /\left(c_{s} k^{2} \lambda_{D}^{2}\right)$ and compare it with the nonlinear time $\tau_{n l} \simeq 1 /(k \delta u)$, the ratio being

$\frac{\tau_{n l}}{\tau_{d}} \simeq \frac{\left(\mathrm{k} \lambda_{D}\right)^{2}}{2 \pi} \frac{c_{s}}{\delta u}$.

For the values of $\mathrm{k} \lambda_{D} \simeq 0.1$ to 0.6 obtained in Sect. 4.2, this ratio is typically of the order of 10 to 100 , so that the dispersive effects are much stronger than the macroscopic nonlinear effects. Since the wave forms shown in Fig. 1 are manifestly affected by nonlinear terms, this indicates that the corresponding source of nonlinearity is probably of microscopic origin.

The second result of our study is that the type of the e.s. waves depends on the proton temperature and on the direction of the magnetic field. This result can be placed in a proper perspective if one recalls that the solar wind speed, density and proton temperature, and 
the magnetic field structure, are strongly dependent on the heliomagnetic latitude. Regions of high proton temperature and with more radial magnetic fields are likely to be found in the fast solar wind, at relatively high heliomagnetic latitudes. Away from the heliospheric current sheet, deep into the fast wind, LF wave packets are observed less and less frequently, and the e.s. activity occurs mainly in the form of WDL.

It is worthwhile mentioning that a number of properties of the particle distribution functions depend also on the distance from the heliospheric current sheet, which explains why good correlations are usually found between IAC wave activity and non Maxwellian features of the distribution functions. For example, suprathermal electrons usually display an asymmetric structure in velocity space called a strahl (see Marsch, 1991, Sect. 8.2.3; Pilipp et al., 1987), a kind of beam more or less loosely aligned with the magnetic field and directed towards the Earth. Pilipp et al. (1987) noticed that the strahl disappears just at the magnetic sector boundary, and that the angular distribution of the strahl around the magnetic field is narrower far from the heliospheric current sheet. It would be interesting to correlate the three states of e.s. activity (wave packets, weak double layers, no e.s. activity) to the three types of electron distributions observed by Pilipp et al. (1987) (broad strahl, narrow strahl, isotropic). This correlation will be studied in a future work.

We want to stress that while $T_{p}$ appears to be an important parameter for the properties of the LF waves, the electron temperature $T_{e}$ has no influence on this electrostatic activity, so that $T_{p} / T_{e}$ is less important than $T_{p}$. Indeed, in our data set, the type of the wave forms depends essentially on $T_{p}$, as discussed in Sect. 3 and 6.2; and waves are observed in the whole range $0.3 \leq$ $T_{e} / T_{p}<4.5$. What can be the influence of the TDS data selection on this result? We made a comparison (not shown here) of the histogram of $T_{p} / T_{e}$ at times when the TDS transmitted a natural wave form, and of the corresponding histogram for all the 3D-Plasma points every $1.5 \mathrm{~min}$ during the considered 38 days: these histograms are very similar, with the same extrema $T_{p} / T_{e}=0.2$ and $T_{p} / T_{e} \simeq 3.5$; the first histogram peaks around 0.8 , while the second one peaks around 0.6. Therefore, we do not expect that our limitation to data with a relatively low density is significant in this respect. Our results on the dominant role of $T_{p}$ differ from the results of Gurnett et al. (1979) who gave evidence for an influence of $T_{e} / T_{p}$. Actually, this contradiction is only apparent since there is a strong anticorrelation between $T_{e} / T_{p}$ and $T_{p}$, partly due to the fact that the range of variation of $T_{e}$ is much smaller than that of $T_{p}$. This anticorrelation is displayed in Fig. 11, where the points correspond to all the TDS events, including artefacts, i.e. are typical of the solar wind at $1 \mathrm{UA}$.

\section{Conclusion}

The main results of the present analysis can be summarised as follows. When the density is smaller than about $10 \mathrm{~cm}^{-3}$, at the Lagrange point $L_{1}$, the time

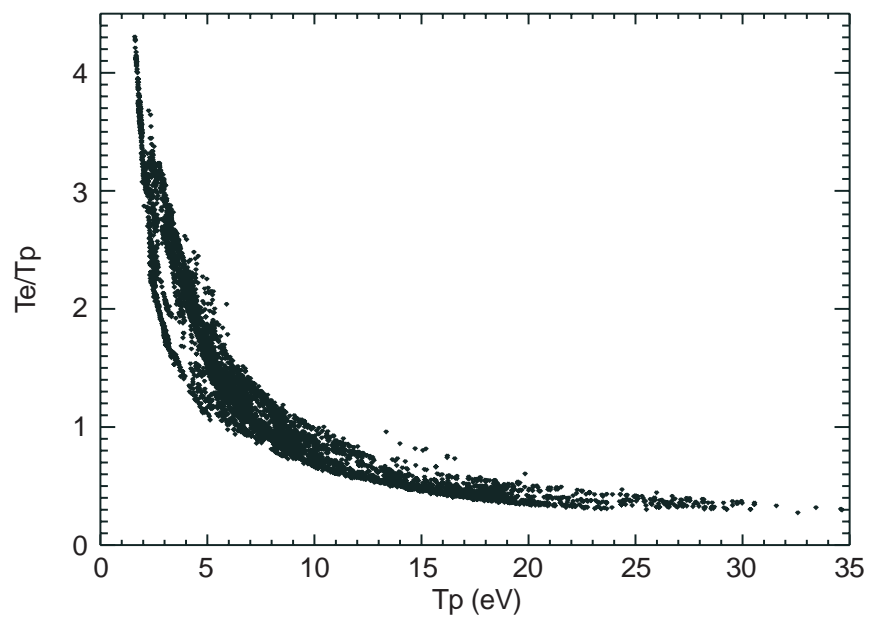

Fig. 11. Scatter plot of $T_{e} / T_{p}$ as a function of $T_{p}$ in $\mathrm{eV}$, measured at the TDS sampling times (natural events plus artefacts)

domain sampler experiment detects a very bursty, but more or less continuous electrostatic activity in the frequency range between the ion and the electron plasma frequency, $f_{p i} \leq f<f_{p e}$. As seen with the TDS, this electrostatic activity appears to be a mixture of coherent wave packets and weak double layers. A typical thickness of the double layers is $25 \lambda_{D}$; and a typical value for the electric potential drop across the double layers is only a few $\mathrm{mV}$, with $q \Delta \Phi /\left(k_{B} T_{e}\right) \simeq 3 \times 10^{-4}$, generally earthward. (When the solar wind density is larger than $10 \mathrm{~cm}^{-3}$ an artefact prevents any observation of the TDS activity.)

The highest intensity and level of wave activity in the range of frequency $f_{p i} \leq f<f_{p e}$ is found for relatively low proton temperatures, at low heliomagnetic latitudes. Towards higher latitudes, the frequency of occurrence of wave packets decreases, and one observes mainly weak double layers deep into the fast solar wind. No clear relation with important plasma parameters like the electron to proton temperature ratio was found.

To conclude, let us note that most of the Langmuir waves observed by the TDS are not associated with type III bursts and appear to be distributed as the low frequency activity described. Their detailed study will be the object of another investigation.

Acknowledgements. The WAVES instrument on WIND was built by teams at the University of Minnesota, the University of Iowa and the Observatoire de Paris, Meudon, with support of NASA/ GSFC. M. L. Kaiser is the Deputy PI. Use of the key parameter MFI data and 3D-plasma data is courtesy of the teams of the Magnetic Field Investigation experiment (PI R.P. Lepping) and the Three Dimensional Plasma experiment (PI R.P. Lin), and of the ISTP CDHF team at NASA/GSFC. The French contribution is supported by the Centre National d'Etudes Spatiales and the Centre National de la Recherche Scientifique.

Topical Editor R. Schwenn thanks E. Marsch and another referee for their help in evaluating this paper.

\section{References}

Bale, S. D., D. Burgess, P. J. Kellogg, K. Goetz, R. L. Howard, and S. J. Monson, Phase coupling in Langmuir wave packets: 
possible evidence of three-wave interactions in the upstream solar wind, Geophys. Res. Lett., 23, 109-112, 1996.

Bale, S. D., D. Burgess, P. J. Kellogg, K. Goetz, and S. J. Monson, On the amplitude of intense Langmuir waves in the terrestrial electron foreshock, J. Geophys. Res., 102, 11,281-286, 1997.

Borovsky, J. E., A review of plasma double-layer simulations, in Second Symposium on Plasma Double Layers and Related Topics, Eds. R. Schrittwieser and G. Eder, Innsbruck, pp. 3354, 1984.

Boström, R., G. Gustafsson, B. Holback, G. Holmgren, H. Koskinen, and P. Kintner, Characteristics of solitary waves and weak double layers in the magnetospheric plasma, Phys. Rev. Lett., 61, 82, 1988.

Bougeret, J. -L., M. L. Kaiser, P. J. Kellogg, R. Manning, K. Goetz, S. J. Monson, N. Monge, L. Friel, C. A. Meetre, C. Perche, L. Sitruk, and S. Hoang, WAVES: the radio and plasma wave investigation on the WIND spacecraft, Space Sci. Rev, 71, 231263, 1995.

Dum, C. T., E. Marsch, W. G. Pilipp, and D. A. Gurnett, Ion sound turbulence in the solar wind, in Solar Wind Four, pp. 299-304, 1981.

Fälthammar, C. G., Laboratory and space experiments as a key to the plasma universe, in Symposium on Plasma-93, Allahabad, India, 1993.

Forslund, D. W., Instabilities associated with heat conduction in the solar wind and their consequences, J. Geophys. Res., 75, 1728, 1970.

Gary, S. P., Ion-acoustic-like instabilities in the solar wind, J. Geophys. Res., 83, 2504-2510, 1978.

Gary, S. P., Theory of space plasma microinstabilities, Cambridge University press, 1993.

Gurnett, D. A., Waves and instabilities, in Physics of the inner heliosphere II, Eds. R. Schwenn and E. Marsch, SpringerVerlag, Berlin Heidelberg New York, pp. 135-157, 1991.

Gurnett, D. A., and R. R. Anderson, Plasma wave electric fields in the solar wind: initial results from Helios 1, J. Geophys. Res., 82, 632-650, 1977.

Gurnett, D. A., and L. A. Frank, Ion acoustic waves in the solar wind, J. Geophys. Res., 83, 58-74, 1978.

Gurnett, D. A., E. Marsch, W. Pilipp, R. Schwenn, and H. Rosenbauer, Ion acoustic waves and related plasma observations in the solar wind, J. Geophys. Res., 84, 2029-2038, 1979.

Kellogg, P. J., S. J. Monson, K. Goetz, R. L. Howard, J. -L. Bougeret, and M. L. Kaiser, Early Wind observations of bow shock and foreshock waves, Geophys. Res. Lett., 23, 1243-1246, 1996.

Kojima, H., H. Matsumoto, T. Miyatake, I. Nagano, A. Fujita, L. A. Frank, T. Mukai, W. R. Paterson, Y. Saito, S. Machida, and R. R. Anderson, Relation between electrostatic solitary waves and hot plasma flow in the plasma sheet boundary layer: GEOTAIL observations, Geophys. Res. Lett., 21, 2919-2922, 1994.

Kurth, W. S., D. A. Gurnett, and F. L. Scarf, High-resolution spectrograms of ion acoustic waves in the solar wind, J. Geophys. Res., 84, 3413-3419, 1979.

Lemons D. S., J. R. Asbridge, S. J. Bame, W. C. Feldman, S. P. Gary and J. T. Gosling, The source of electrostatic fluctuation in the solar wind, J. Geophys. Res., 84, 2135, 1979.

Lepping, R. P., M. H. Acuña, L. F. Burlaga, W. M. Farrell, J. A. Slavin, K. H. Schatten, F. Mariani, N. F. Ness, F. M. Neubauer, Y. C. Whang, J. B. Byrnes, R. S. Kennon, P. V. Panetta, J. Scheifele, and E. M. Worley, The Wind magnetic field investigation, in The global Geospace mission, Ed. C.T. Russell, Kluwer Academic Publishers, pp. 207-229, 1995.

Lin, N., P. J. Kellogg, R. J. MacDowall, A. Balogh, R. J. Forsyth, J. L. Phillips, A. Buttighoffer, and M. Pick, Observations of plasma waves in magnetic holes, Geophys. Res. Lett., 22, 34173420, 1995a.

Lin, R. P., K. A. Anderson, S. Ashford, C. Carlson, D. Curtis, R. Ergun, D. Larson, J. McFadden, M. McCarthy, G. K. Parks, H. Rème, J. M. Bosqued, J. Coutelier, F. Cotin, C. d'Uston, K.-P. Wenzel, T. R. Sanderson, J. Henrion, J. C. Ronnet, and G. Paschmann, A three-dimensional plasma and energetic particle investigation for the Wind spacecraft, in The global Geospace mission, Ed. C.T. Russell, Kluwer Academic Publishers, pp. 125-153, 1995b.

MacDowall, R. J., R. A. Hess, N. Lin, G. Thejappa, A. Balogh, and J. L. Phillips, Ulysses spacecraft observations of radio and plasma waves: 1991-1995, Astron. Astrophys., 316, 396-405, 1996.

Mälkki A., Eriksson A. I., Dovner P., R. Boström, B. Holback, G. Holmgren, and H. E. J. Hoskinen, A statistical study of auroral solitary waves and weak double layers, 1 . Occurrence and net voltage, J. Geophys. Res., 98, 15521, 1993.

Marsch, E., Beam-driven electron acoustic waves upstream of the Earth's bow shock, J. Geophys. Res., 90, 6327-6336, 1985.

Marsch, E., Kinetic physics of the solar wind plasma, in Physics of the inner heliosphere II, Eds. R. Schwenn and E. Marsch, Springer-Verlag, Berlin Heidelberg New York, pp. 45-133, 1991.

Matsumoto, H., H. Kojima, T. Miyatake, Y. Omura, M. Okada, I. Nagano, and M. Tsutsui, Electrostatic solitary waves (ESW) in the magnetotail: BEN wave forms observed by GEOTAIL, Geophys. Res. Lett., 21, 2915-2918, 1994.

Meetre, C., K. Goetz, R. Manning, J. -L. Bougeret, and C. Perche, A realtime neural network in space, Lund, July 29-31, 1997, in press in ESA Publications, 1998.

Meyer-Vernet, N., and C. Perche, Toolkit for antennae and thermal noise near the plasma frequency, J. Geophys. Res., 94, 24052415, 1989.

Mottez, F., S. Perraut, A. Roux, and P. Louarn, Coherent structures in the magnetotail triggered by counterstreaming electron beams, J. Geophys. Res., 102, 11399-11408, 1997.

Mozer, F. S., and M. Temerin, Solitary waves and double layers as the source of parallel electric fields in the auroral acceleration region, in High Latitude Space Plasma Physics, Eds. B. Hultqvist and T. Hagfors, Plenum, London, 44, pp. 453, 1983.

Omura, Y., H. Kojima, and H. Matsumoto, Computer simulation of electrostatic solitary waves: a nonlinear model of broadband electrostatic noise, Geophys. Res. Lett., 21, 2923-2926, 1994.

Omura, Y., H. Matsumoto, T. Miyake, and H. Kojima, Electron beam instabilities as generation mechanism of electrostatic solitary waves in the magnetotail, J. Geophys. Res., 101, 26852697, 1996.

Pilipp, W. G., H. Miggenrieder, K. -H. Mulhauser, H. Rosenbauer, R. Schwenn, and F. M. Neubauer, Variations of electron distribution functions in the solar wind, J. Geophys. Res., 92, 1103-1118, 1987.

Richaume, P., J. -L. Bougeret, C. Perche, and S. Thiria, Simulation des traitements réseaux de neurones embarqués sur la sonde spatiale Wind/Waves, in Neural networks and their applications 1994, pp. 141-150, 1995.

Sanderson, T. R., R. P. Lin, D. Larson, M. P. McCarthy, G. K. Parks, J. M. Bosqued, N. Lormant, K. Ogilvie, R. P. Lepping, A. Szabo, A. J. Lazarus, J. Steinberg, and J. T. Hoeksema, Wind observations of the influence of the Sun's magnetic field on the interplanetary medium at 1 AU, J. Geophys. Res., 103, 17235 $17247,1998$.

Temerin, M., K. Cerny, W. Lotko, and F. S. Mozer, Observations of double layers and solitary waves in the auroral plasma, Phys. Rev. Lett., 48, 1175-1179, 1982.

Treumann, R. A., and W. Baumjohann, Advanced Space Plasma Physics, Imperial College Press, 1997. 